Revue d'histoire de l'Amérique française

RAS REVUE D.HISTOIRE DE L'AMÉRIQUE FRANÇAISE

\title{
Nouvelles perspectives sur l'histoire sociale du Québec
}

\section{Fernand Harvey}

Volume 24, numéro 4, mars 1971

URI : https://id.erudit.org/iderudit/303020ar

DOI : https://doi.org/10.7202/303020ar

Aller au sommaire du numéro

Éditeur(s)

Institut d'histoire de l'Amérique française

ISSN

0035-2357 (imprimé)

1492-1383 (numérique)

Découvrir la revue

Citer cette note

Harvey, F. (1971). Nouvelles perspectives sur l'histoire sociale du Québec.

Revue d'histoire de l'Amérique française, 24(4), 567-581.

https://doi.org/10.7202/303020ar d'utilisation que vous pouvez consulter en ligne.

https://apropos.erudit.org/fr/usagers/politique-dutilisation/ 


\title{
NOTES CRITIQUES
}

I

\section{NOUVELLES PERSPECTIVES SUR L'HISTOIRE SOCIALE DU QUEEBEC}

Quelques thèses récentes présentées à

l'Institut d'Histoire de l'Université Laval

\author{
Fernand Harvey \\ Institut supérieur des \\ sciences humaines \\ Université Laval
}

L'histoire sociale est demeurée longtemps le parent pauvre de l'historiographie québécoise. Il aura fallu attendre les travaux de Fernand Ouellet ${ }^{1}$, Jean Hamelin ${ }^{2}$ et Marcel Trudel pour que s'instaure une véritable tradition en histoire socio-économique au Québec. Depuis lors, les travaux de Cameron Nish ${ }^{3}$, Michel Têtu ${ }^{4}$, Jean-Pierre Wallot, Gilles Paquet ${ }^{5}$ et de plusieurs autres ${ }^{6}$ sont venus enrichir ce courant. Parallèlement à ces travaux, on compte plusieurs monographies sur l'idéologie économique et sociale de certains hommes publics, de même que sur certains journaux ou revues de diverses tendances ? . Ces analyses d'idéo-

\footnotetext{
1 Fernand Ouellet, Histoire économique et sociale du Québec 1760-1850 (Montréal, Fides, 1966).

2 Jean Hamelin, Economie et Société en Nouvelle-France (Québec, Presses de l'Université Laval, 1960).

3 Cameron Nish, Les bourgeois-gentilhommes en Nouvelle-Francs (Montréal, Fides, 1968).

4 Michel Têtu, Les premiers syndicats catholiques canadiens, 19001921. Doctorat (Lettres), (Université Laval, 1961).

5 Gilles Paquet et Jean-Pierre Wallot, "La liste civile du Bas-Canada (1794-1812): un essai d'économie historique", RHAF (23 : 209-230; 361-392 et $24: 3-43$.

6 Parmi ceux-ci citons : Denys Delage, Canada et New-York, 16081750. Thèse de maîtrise (Sociologie), (Université de Montréal, 1969), $277 \mathrm{p}$. et Gilles Bourque, Classes sociales et question nationale au Québec, 17601840. Thèse de maîtrise (Sociologie), (Université de Montréal, 1969), $284 \mathrm{p}$. (Publiée aux éditions Parti Pris en 1970).

7 Voir à ce sujet "Idéologies au Canada français 1850-1900", Recherches sociologiques, $\mathrm{X}: 2-3$ (mai-déc. 1969) : $491 \mathrm{p}$. On pourra aussi trouver une liste courante des travaux sur les idéologies et l'histoire sociale dans la bibliographie trimestrielle de la RHAF.
} 
logies, bien que présentant un intérêt évident pour la connaissance du Québec, demeureront limitées aussi longtemps qu'elles n'auront pas été complétées par une analyse correspondante de la structure sociale.

Quelques thèses récentes en histoire sociale présentées à l'Institut d'histoire de l'Université Laval apportent une contribution d'importance à la connaissance de la société québécoise du XIX $\mathrm{XI}^{\mathrm{e}}$ siècle et des débuts du $\mathrm{XX}^{\mathbf{e}}$ siècle. L'histoire sociale du Québec était demeurée jusqu'ici un appendice de l'histoire économique. Pour la première fois, nous semble-t-il, un véritable courant autonome en histoire sociale est en voie d'élaboration. Il convient ici de souligner le rôle d'animateur du professeur Jean Hamelin dans l'orientation et la formation d'une nouvelle génération d'historiens qui se consacrent à l'étude des structures sociales et des mouvements sociaux.

Entreprendre la critique de thèses de maîtrise constitue une tâche délicate. Les auteurs critiqués en sont à leur premier essai et leurs travaux comportent des faiblesses inévitables. Nous croyons néanmoins que la rédaction quasi-simultanée du résultat de ces recherches valait la peine d'être soulignée.

David Terence Ruddell, dans une thèse sur l'apprentissage au Québec, entre 1793 et $1815^{8}$, s'applique à dégager les structures et l'évolution d'un certain nombre de métiers au niveau de l'apprentissage. Utilisant comme documentation de base les greffes des notaires de l'époque, il s'attache plus spécifiquement à l'étude de sept métiers : les boulangers, les forgerons, les cordonniers, les charpentiers de navire, les tailleurs, les menuisiers et les tonneliers.

Un premier coup d'œil révèle l'absence d'uniformité quant aux normes qui régissent ces métiers. Le temps d'apprentissage, par exemple, varie sensiblement d'un métier à l'autre et la vie de l'apprenti dépend de sa relation avec son maître et de la nature du travail du maître. L'apprenti, par ailleurs, se distingue nettement du journalier ou du serviteur par la mobilité qu'il peut espérer, contrairement à ces derniers qui n'ont pratiquement aucune chance d'avancement (p. 4). Pour avoir comparé les conditions d'apprentissage au Québec avec celles qui existent en France, en Angleterre et en Nouvelle-Angleterre, Ruddell

8 David Terence Ruddell, Apprenticeship in early nineteenth century Quebec, 1793-1815. Thèse de M.A. (Histoire), (U. Laval, nov. 1969), 190 p. 
en vient à la conclusion qu'il n'existe pas de différences fondamentales sur le plan de la hiérarchie de travail et de la forme de contrat entre la France et la Nouvelle-France ni entre le Québec anglophone et l'Angleterre ou la Nouvelle-Angleterre (p. 15).

Dans un second chapitre, Ruddell s'efforce de situer l'artisan et l'apprenti dans son milieu social. L'artisan se fixerait surtout dans une zone urbaine ou à proximité des deux principales villes du Québec : Montréal et Québec. L'économie intérieure du BasCanada à la fin du XVIII ${ }^{e}$ siècle présente, selon l'auteur, un caractère autarcique. Dans un tel contexte, l'industrie familiale polyvalente suffit aux besoins des régions rurales. Les relations économiques entre les campagnes et les villes se réduisent à l'achat par l'agriculteur de quelques produits, tels l'eau-de-vie, la mélasse, le tabac, l'huile, en échange de produits agricoles ou de viande de boucherie (p. 33-34). A la fin du $18^{\mathrm{e}}$ siècle, note Ruddell, le commerce intérieur continue de se caractériser par des commandes personnelles et une production artisanale. Un individu a-t-il besoin d'une paire de souliers ou de se faire construire une maison qu'il en fait la demande à un artisan qui se charge d'exécuter la commande avec l'aide de quelques compagnons et apprentis, souvent des membres de sa propre famille (p. 39).

Ruddell souligne également l'impact de la conjoncture économique entre 1793 et 1815 sur l'évolution des métiers. L'expansion des exportations de bois vers la Grande-Bretagne à l'occasion de la guerre de 1812 contribue au développement de certains métiers et, par voie de conséquence, à l'augmentation du nombre d'apprentis (p. 54a).

Comme nous l'avons relevé au début, les normes qui régissent l'apprentissage varient d'un métier à l'autre. Il semble cependant que les heures de travail des apprentis-artisans, si l'on excepte les domestiques, sont à peu près de 11 heures par jour l'hiver et de 12 heures par jour l'été. L'apprenti est souvent astreint par contrat à exécuter, en plus de son travail régulier, certains travaux domestiques le soir. Règle générale le maître s'engage à fournir l'habillement, le gîte et la nourriture à ses apprentis. Certains maîtres prennent cependant l'habitude d'octroyer à leurs apprentis une allocation en argent qui leur permet de se dégager de cette obligation ( $p .118$ ).

Abordant dans un dernier chapitre les conditions sociales de l'apprentissage, Ruddell établit certaines constatations:

1 - Les apprentis et leurs parents viennent de la campagne et 
de la ville et les parents de l'apprenti sont surtout des artisans ou des habitants.

2 - La majorité des apprentis ruraux viennent des villages et des paroisses environnantes; quant à ceux des villes, ils sont issus de différents quartiers ou de la banlieue.

3 - Il existe peu de perméabilité entre le groupe des professionnels et des marchands et celui des artisans (p. 139).

L'analyse de Ruddell nous met en présence d'une structure sociale relativement rigide où la mobilité sociale apparaît comme un phénomène d'exception. La classe des marchands et celle des professionnels défendent leur statut social en favorisant leurs enfants, au niveau de l'apprentissage, et en empêchant les fils d'artisans, de fermiers, de soldats, ou de journaliers, d'accéder à ces professions. Le même phénomène peut être observé chez les artisans, face aux fils de soldats et de journaliers (p. 144). En somme, l'occupation du père est déterminante pour le choix, par le fils, d'un métier ou d'une professioñ. Il est intéresssant de noter par ailleurs, qu'en dépit d'obstacles réels, les barrières ethniques n'apparaissent pas comme infranchissables. Il existe une coopération relative entre marchands et professionnels canadiens-anglais et canadiens-français "who appeared to cooperate with others, in accepting, as apprentices, the sons of the ethnic group" (p. 143).

Ruddell souligne enfin que la pratique religieuse des apprentis est un souci important pour les parents francophones et catholiques alors que le problème ne semble pas se poser pour les parents anglophones et protestants. Ces derniers, par contre, se préoccupent davantage d'assurer à leurs fils une éducation théorique.

La recherche de Ruddell soulève de nombreuses hypothèses nouvelles. L'auteur a conscience du caractère provisoire de ses conclusions. Sa contribution à la connaissance de la société québécoise du début du XIX ${ }^{\circ}$ siècle mérite cependant d'être soulignée. Sa méthode d'analyse est à la fois prudente et solide: ses affirmations sont toujours appuyées sur une documentation sûre. Il faut souhaiter que l'auteur poursuive ses recherches dans cette voie et pousse plus avant au niveau de l'interprétation. Ruddell dégage, en effet, une série de découvertes très intéressantes, mais sa trop grande prudence d'historien l'empêche d'avancer certaines hypothèses qui semblent s'imposer, au niveau de la structure sociale. 
La thèse de Jacques Rouillard porte sur les filatures de coton au Québec entre 1900 et $1915^{\circ}$. L'auteur s'appuie sur une documentation solide et variée. Il a consulté principalement les archives de deux commissions royales d'enquête sur l'industrie textile (1908 et 1938), de nombreuses publications gouvernementales, des journaux dont La Presse et La Patrie et un certain nombres d'études.

Le premier chapitre est consacré à l'étude de l'économie de l'industrie textile du coton. L'auteur souligne à juste titre que la période qu'il étudie coïncide avec une concentration de plus en plus poussée de l'industrie textile au Québec ( $p$. 10). Cette concentration avait été précédée par le développement d'une multitude de petites et de moyennes manufactures de textiles dont la fondation s'était échelonnée tout au cours de la seconde moitié du $\mathrm{XIX}^{\mathrm{e}}$ siècle et principalement durant les années 1880 . La multiplicité des manufactures, encouragée par la politique nationale de Macdonald, avait abouti à une surproduction et à une crise à la fin $d u X X^{e}$ siècle. La fondation, après plusieurs tentatives infructueuses, de la Dominion Textile, en 1905, permettra une relance de l'industrie du coton au Québec. Le nouveau trust bénéficiait de prix relativement élevés, d'une augmentation de la consommation et de l'ouverture du marché de l'Ouest canadien (p. 29).

A l'aide de tableaux et de graphiques, Rouillard tente de préciser le degré de prospérité de l'industrie textile en établissant la variation des dividendes payés aux actionnaires et la variation du prix du produit fini. Il conclut que, pour la période 1908-1915, l'industrie textile manifeste des signes de prospérité et il attribue cet essor à la hausse des prix, rendue possible grâce au contrôle du marché par la Dominion Textile (p. 54).

Abordant la question des facteurs de régionalisation, Rouillard se refuse à une explication univoque. Pour lui, plusieurs facteurs ont facilité l'implantation de l'industrie textile au Québec. Parmi ceux-ci, citons : la main-d'œuvre plus abondante et moins coûteuse qu'ailleurs, l'énergie hydraulique, l'axe de chemin de fer Montréal-Portland, et l'esprit d'entreprise de certains industriels. L'auteur souligne au passage l'absence quasitotale des Canadiens français aux postes de commande. A Valleyfield par exemple, il faudra attendre jusqu'en 1900 pour qu'un Canadien français soit promu au poste d'assistant-contremaître...

9 Jacques Rouillard, Les filatures de coton au Québec, 1900-1915. Thèse de M.A. (Histoire), (Université Laval, sept. 1970), 176 p. 
Le travailleur du textile fait l'objet du second chapitre dt cette recherche. Avec le début du $\mathrm{XX}^{\circ}$ siècle, la composition de la main-d'œuvre subit des changements importants. Le pourcentage de femmes composant la main-d'œuvre totale des filatures au Québec passe de $70 \%$ en 1870 à $42 \%$ en 1915 . L'évolution technique serait à la base du déclin du nombre d'employés féminins dans les filatures (p. 71). Par ailleurs, le pourcentage de la main-d'œuvre infantile travaillant dans les filatures au Québec est supérieur à celui de l'étranger. Rouillard explique ce phénomène par l'esprit de coopération qui animerait la famille canadienne-française rurale traditionnelle. Cette solidarité organique de la famille inciterait les parents à faire travailler leurs enfants (p. 73-74).

L'absence de législation effective touchant l'âge minimum d'entrée sur le marché du travail, avant 1907, encourage les enfants à travailler dans les filatures. "Le travail des enfants, écrit Rouillard, engendre une diminution des salaires qui, à son tour, force les pères de famille à entraîner leurs enfants à la manưfacture." (p. 77) Le travail des enfants, poursuit l'auteur, "accentue l'analphabétisme et, à long terme, constitue un prolétariat canadien-français incapable d'occuper des postes de commande" (p. 79).

Au problème du travail des enfants s'ajoute celui des longues heures de travail. En 1888, nombreux sont les femmes et les enfants qui dépassent les 60 heures par semaine fixées par la loi de 1885 . Il faudra attendre la loi de 1912 pour voir réduire à 55 heures, la semaine de travail (p. 81).

D'autres problèmes contribuent à accentuer la condition déjà pénible des travailleurs du coton : hygiène déficiente, contremaîtres brutaux et arbitraires, insécurité d'emploi, danger des accidents de travail, absence d'assurance ou d'indemnisation par suite d'un accident de travail, etc.

Grâce à l'action des syndicats et à une période de prospérité, les salaires augmentent de $25 \%$ de 1906 à 1908. "Cependant, en mai 1908, la Dominion Textile et la Montreal Cotton décrètent une diminution générale des gages de 10\%." (p. 93) Ce type de situation qu'on retrouve aussi dans d'autres industries au XIX' et au début du $\mathrm{XX}^{\mathrm{e}}$ siècle nous révèle la faiblesse du mouvement syndical de l'époque et la toute-puissance des entreprises au niveau des relations de travail.

Dans un troisième chapitre consacré au syndicalisme dans l'industrie textile, Rouillard distingue deux causes qui explique- 
raient la difficulté des chefs syndicaux à constituer une force syndicale bien structurée dans l'industrie textile. "En premier lieu, écrit-il, les filatures emploient une forte proportion de femmes et d'enfants difficilement syndicables." ( $p .103$ ) "D'autre part, les filatures requièrent en majorité l'emploi d'une maind'œuvre peu expérimentée ou à demi-spécialisée que les manufacturiers remplacent facilement en cas de grève. Leurs bas salaires ne les incitent pas non plus à risquer de le perdre dans une aventure comme le syndicalisme." (p. 104)

Ce chapitre sur le syndicalisme dans l'industrie textile est sans contredit le plus intéressant et le plus personnel de toute la recherche de Rouillard. L'auteur analyse l'évolution du syndicalisme en fonction de la variable technique. Il constate la faiblesse de la position de négociation des travailleurs du textile, due à leur situation d'ouvriers non-spécialisés. C'est pourquoi les ouvriers du textile se sentent plus à l'aise à l'intérieur des Chevaliers du travail qui proposent une stratégie globale pour la classe ouvrière, contrairement aux unions de métiers qui ne s'intéressaient qu'à la promotion économique de leurs membres (p. 109). Après la disparition des Chevaliers du travail au tout début du $\mathrm{XX}^{\mathrm{e}}$ siècle, les travailleurs du textile s'efforcent tant bien que mal de s'intégrer à divers syndicats de métiers.

Une autre hypothèse de Rouillard mérite d'être relevée. S'appuyant sur une étude du sociologue Alain Touraine sur les ouvriers d'origine agricole (1961), l'auteur émet l'hypothèse que les ouvriers canadiens-français d'origine agricole transplantés en milieu urbain ont du mal à adopter des attitudes ouvrières dès la première génération. Satisfaits d'une mobilité sociale réussie, ils constituent une main-d'œuvre docile (p. 105-6). Il faudra attendre la deuxième génération d'ouvriers, celle née à la ville, pour assister à la naissance d'une véritable conscience ouvrière (p. 107).

Rouillard termine sa recherche par une analyse des grèves dans le textile. Celles-ci sont nombreuses puisque le secteur du textile se classe au troisième rang quant aux jours ouvrables perdus, entre 1901 et 1915. Avant la fondation de la Fédération des Ouvriers textiles du Canada, en 1906, ce sont les élites locales qui, dans des petites villes comme Valleyfield, servaient d'intermédiaires pour régler les conflits de travail entre les ouvriers et les patrons (p. 160). "Les grèves du textile, conclut Rouillard, révèlent un monde ouvrier faible soumis aux aléas de la conjoncture économique, ballotté entre la plus pure soumission et des mouvements brefs et violents de révolte." (p. 164) 
Portant sur la même période que la thèse de Rouillard, celle de Paul Larocque, sur la condition socio-économique des travailleurs de la ville de Québec ${ }^{10}$, se présente dans une perspective assez différente. Larocque cherche à cerner la condition ouvrière à l'intérieur d'une ville et non pas en fonction d'une industrie spécifique. La documentation qui sert de base à cette étude est variée. Les publications gouvernementales, en particulier, sont citées abondamment: Gazette du Travail, rapports d'inspecteurs, recensements, etc.

Après avoir localisé les quartiers ouvriers à l'intérieur de la ville de Québec, Larocque s'emploie à décrire la condition qui prévaut dans ces quartiers. Une constatation évidente se dégage de ce chapitre : l'état embryonnaire des services publics et communautaires. Les spéculateurs profitent de l'absence de plan d'urbanisme; l'hygiène publique est dans un état lamentable. Quant aux règlements de santé publique, ils sont difficilement appliqués à cause d'un personnel d'inspection insuffisant.

Face à un tel environnement urbain, il n'est pas ćtennant de constater l'importance de la mortalité infantile (0-5 ans) qui constitue $44.1 \%$ de tous les décès entre 1895 et 1908. La diarrhée infantile et la tuberculose comptent le plus grand nombre de victimes. La densité de la population, la pollution de l'air, la pauvreté, l'ignorance et des conditions de travail pénibles entretiennent un climat favorable à la maladie.

Larocque consacre un chapitre à la main-d'œuvre. Il constate que les femmes de moins de 25 ans ont tendance à travailler surtout dans les manufactures contrairement à celles de plus de 25 ans qui préfèrent les emplois de domestiques. L'auteur y voit l'annonce d'un conflit de générations, les plus jeunes préférant des heures moins longues et un salaire plus élevé (p. 82). Sur le travail des enfants, les remarques de Larocque sont sensiblement les mêmes que celles de Rouillard. En dépit de la législation sur le travail des enfants qui s'échelonne de 1885 à 1907 , de nombreuses fraudes continuent de subsister, à cause du refus des enfants d'aller à l'école, de l'hostilité des parents face à la scolarisation et du besoin de gagner pour la famille (p. 88). Si la lutte contre l'analphabétisme marque des points entre 1901 et 1911, la majorité cependant ne dépasse pas le cours primaire, lequel forme surtout des commis et non des ouvriers qualifiés (p. 96).

10 Paul Larocque. La condition socio-économique des travailleurs de la ville de Québec (1896-1914). Thèse de M.A. (Histoire), (Université Laval, 1970), $212 \mathrm{p}$. 
Après avoir passé en revue diverses questions relatives à la main-d'œuvre, Larocque conclut:

En dépit du progrès notable des conditions de travail, favorisé par la diminution des heures de labeur et les améliorations hygiéniques dans les manufactures, une insécurité occupationnelle notoire subsiste malgré quelques mesures tardives, telles que la loi sur les accidents de travail en 1909 ou la fondation d'un bureau provincial de placement en 1911. Et si le travail de la femme ou de l'adolescent contribue à atténuer l'inquiétude de plusieurs chefs de famille, l'on ne peut en contrepartie qu'évoquer la persistance d'une barrière cristallisant plus nettement qu'aujourd'hui les diverses couches sociales: le faible degré de scolarisation chez les masses en témoigne, malgré un recul certain de l'analphabétisme (p. 140).

Abordant la question du niveau de vie et de la sécurité sociale, l'auteur constate le bas niveau de revenu du travailleur à gages comparé à celui du travailleur salarié. "Moins nombreux, les salariés constituent, en quelque sorte, une élite qui encadre les autres employés." (p. 150) Quant à l'assistance sociale, c'est par la voie de sociétés privées qu'elle se manifeste. Cette assistance privée ne dispose que de moyens sommaires et tente de parer au plus urgent. Ce palliatif, écrit Larocque, "témoigne à la fois de la quasi-inexistence de l'assistance publique et de la précarité du niveau de vie chez certaines couches de la population" (p. 175).

Le sujet abordé par Larocque était d'envergure. Ne disposant d'aucune monographie sur l'un ou l'autre des aspects étudiés, l'auteur a dû recourir directement aux sources. Ces sources, à peu près inexplorées jusqu'ici, renferment quantité de témoignages et de statistiques sur la condition ouvrière. L'auteur a su utiliser ces sources abandamment pour bien illustrer la complexité des divers facteurs qui composent la vie ouvrière de l'époque. Il aurait cependant eu intérêt à restreindre davantage son sujet, vu l'abondance des sources. L'unité et la densité du texte en auraient été renforcées. Il n'en demeure pas moins que cette thèse constitue un travail de défrichage considérable et qu'elle servira de point de repère pour de futurs chercheurs intéressés à approfondir certains points particuliers.

$\mathrm{Au}$ moment où nous écrivons ces lignes, nous n'avons pu prendre connaissance de la thèse de Jacques Bernier sur la condi- 
tion ouvrière dans la ville de Montréal à la fin du XIX $\mathrm{XI}^{\mathbf{e}}$ siècle ${ }^{11}$. Son tuteur, M. Jean Hamelin, nous informe que le cadre d'analyse de Bernier présente des analogies avec celui de Larocque. Bernier s'est cependant intéressé plus particulièrement aux groupes inarticulés et marginaux de la société qui n'ont pas retenu l'attention des historiens québécois jusqu'ici (alcooliques, prostituées, etc).

La thèse de Jacques-P. Mathieu est consacrée à l'étude de la condition ouvrière dans l'industrie du cuir à Québec, de 1900 à $1930^{12}$. Comme le titre l'indique, l'auteur utilise comme source principale les procès-verbaux d'un syndicat. Cette méthode comporte des inconvénients vu la documentation restreinte. Il devient, en effet, difficile de pondérer les jugements émis, l'absence de sources complémentaires ne permettant pas d'appuyer ou de rejeter certaines affirmations.

Une étude des conditions matérielles de l'industrie de la chaussure amorce la recherche. Mathieu fournit des renseignements intéressants sur les étapes de la fabrication des chaussures à la fin du XIX ${ }^{e}$ siècle (p. 7-9). On regrette qu'il n'ait pas poussé plus loin dans cette voie, car cette industrie illustre bien le passage de la division sociale du travail employée dans les sociétés traditionnelles à la division technique du travail mise de l'avant à l'occasion de la seconde révolution industrielle.

La plus grande partie de cette recherche consiste en une analyse de contenu de l'idéologie du syndicat des monteurs. L'aspect national, note l'auteur, occupe un poste important dans leur vision de la société, du fait de leur adhésion à l'idéologie ultramontaine liant religion et nation. "Mais en pratique, il semble bien que leur vie syndicale passe avant leur vie nationale." (p. 38) Quant à l'action syndicale proprement dite, elle s'inspire directement de l'encyclique Rerum Novarum de Léon XIII. La dimension religieuse, d'une façon générale, semble jouer un grand rôle dans la formation des syndiqués monteurs. A partir de 1922, une retraite fermée est même organisée à leurs intentions. Dans un tel contexte, l'aumônier joue un rôle de premier plan. Mathieu laisse entendre qu'il serait un "temporisateur" et un allié indirect des patrons (p. 50-52). Il est ce-

11 Jacques Bernier, La condition ouvrière à Montréal à la fin du XIXe siècle (1774-1896). Thèse de M.A. (Histoire), (Université Laval).

12 Jacques-P. Mathieu, La condition ouvrière dans l'industrie du cuir à Québec, de 1900 à 1930 , d'après les procès-verbaux d'un syndieat. Thèse de M.A. (Histoire), (Université Laval, septembre 1969), 120 p. 
pendant difficile d'accepter une généralisation de ce jugement à cause des sources restreintes dont disposait l'auteur. Il faudra attendre d'autres études sur le rôle des aumôniers dans les syndicats catholiques pour vérifier ce jugement.

Sur le plan politique, la participation des syndiqués, au dire de Mathieu, ne s'exprime qu'au niveau individuel:

De tous ces témoignages, il ressort que les ouvriers considèrent comme important le système politique et qu'ils veulent s'y conformer sans suivre l'exemple des ouvriers révolutionnaires, c'est-à-dire sans viser la prise du pouvoir par les forces ouvrières et sans exiger des changements globaux (p. 69).

Au niveau des relations avec les autres syndicats, les cordonniers monteurs manifestent une "conscience de classe étroite" et rejettent le syndicalisme international (p. 96).

D'une façon générale, la thèse de Mathieu, vu ses limites au niveau des sources, ne saurait présenter de conclusions définitives. Il faudra reprendre les hypothèses émises dans le cadre de recherches plus poussées.

\section{A la recherche d'une méthodologie nouvelle}

Les travaux présentés ci-haut inaugurent, chacun dans leur sphère, des champs de recherches jusqu'ici en friche. Ils marquent, par le fait même, le début d'une tradition en histoire sociale à l'Université Laval. Il était inévitable que ces premières tentatives présentent des difficultés au niveau de la documentation et de la méthodologie. Ces premiers coups de sonde ont prouvé qu'il existe, en dépit de ce que l'on pourrait croire au premier abord, une documentation considérable pour servir à l'étude de l'histoire sociale et en particulier de l'histoire des travailleurs. Encore faut-il avoir la patience de réunir un matériel dispersé dans les journaux, les rapports gouvernementaux et certains fonds d'archives, tels ceux des municipalités. L'expérience acquise par ces premières recherches permettra sans doute une meilleure orientation des futurs chercheurs. Parmi les sujets qui mériteraient d'être étudiés soulignons quelques exemples: l'industrie du cuir au $\mathrm{XIX}^{\mathrm{e}}$ siècle, particulièrement au niveau de son évolution technique; les sociétés de secours mutuel et les sociétés de bienfaisance, les débardeurs de Montréal et de Québec, l'hygiène publique dans les villes, les premiers syndicats catholiques entre 1900 et $1920 \ldots$. 
Ces travaux ont aussi posé le problème de la méthodologie en histoire sociale. Contrairement à l'histoire politique ou à l'histoire des idées qui présentent des champs de recherche relativement bien définis, l'histoire sociale possède des frontières relativement perméables et pourrait, à la limite, englober l'ensemble de la discipline historique. Elle risque, de plus, de s'empêtrer dans une masse considérable de documentation si elle néglige d'élaborer une méthodologie adéquate. Le problème est particulièrement aigu pour la période postérieure aux années 1870. Il importe à l'historien de dépasser ici les cadres de sa méthodologie traditionnelle s'il veut éviter de demeurer prisonnier d'une histoire purement descriptive. Le recours aux sciences sociales et plus particulièrement à la sociologie s'impose comme une nécessité. La perspective interdisciplinaire est admise chez la majorité des historiens. Malheureusement il faut avouer qu'elle est demeurée, la plupart du temps, au niveau des bonnes intentions. On aurait tort de jeter le blâme sur les seuls historiens. Les sociologues, avant tout préoccupés des problèmes actuels, ont longtemps négligé la dimension historique. Le sociologue ou l'histôrieñ cuui désire aborder la question de l'industrialisation du Québec, à la fin du XIX $\mathrm{X}^{\mathrm{e}}$ siècle par exemple, ne dispose pas de modèles bien articulés, à l'exception du modèle marxiste. Cette lacune se manifeste encore avec plus d'évidence lorsqu'il s'agit d'étudier une société traditionnelle non primitive comme la société canadienne du XVIII ${ }^{\mathrm{e}}$ ou du XIX XIècle $^{13}$.

Face à cette impasse méthodologique, l'historien se doit de prendre l'initiative et de pénétrer dans le secteur de la sociologie qui correspond à son champ de recherche. Peut-on concevoir, par exemple, qu'un historien du travail ignore tout des relations industrielles et de la sociologie du travail ? Pour se pénétrer de l'esprit d'une discipline nouvelle, l'historien doit faire l'effort de se décentrer face à sa propre méthode et sa propre perspective. Cette "ascèse méthodologique" ne saurait que lui être bénéfique à long terme. L'historien qui accepte de s'y soumettre ne découvrira pas de modèles faits à l'avance. Sa démarche consistera essentiellement à transposer au passé, en les adaptant, certaines hypothèses et certaines conclusions de recherches récentes. Si l'on accepte de se situer dans la perspective de la "longue durée", selon l'expression de Braudel, ne pouvons-nous pas découvrir certaines constantes à l'intérieur de la société industrielle, et certaines questions étudiées par les sociologues contemporains ne se

${ }^{13}$ La plupart des travaux des anthropologues sur la société traditionnelle concernent des groupes ethniques archaïques ou primitifs et n'offrent guère de modèles pour l'étude des sociétés traditionnelles de type historique. 
trouvent-elles pas déjà à l'état embryonnaire à l'origine de la société industrielle?

La voie comparative s'offre aussi à l'historien qui explore les avenues de la sociologie. En comparant, voire même en opposant un phénomène passé à un phénomène actuel, l'historien sera en mesure de mieux discerner l'essence et les composantes de ce phénomène passé. Il est significatif, à cet égard, de constater que la naissance, au $\mathrm{XIX}^{\mathrm{e}}$ siècle, de l'anthropologie scientifique coïncide avec l'essor de la société industrielle et la perception de la culture traditionnelle comme distance.

La méthode comparative peut être particulièrement utile à l'historien des sociétés traditionnelles en opposant par exemple société traditionnelle et société technique, travail artisanal et travail industriel, corporations de métiers et syndicats, outils traditionnels et machines, etc. En procédant ainsi par couples opposés, l'historien en viendra à se constituer une problématique propre au sujet étudié; ce qui ne l'empêchera pas, bien au contraire, d'utiliser la méthode traditionnelle pour étudier ses sources. C'est chargé de nouvelles questions et de nouvelles hypothèses qu'il interrogera le passé.

Pour la période pré-industrielle, l'histoire sociale pourrait également s'inspirer de travaux effectués en fonction d'aires géographiques différentes ou de périodes chronologiques différentes. Sous bien des aspects, l'artisan en Nouvelle-France n'est-il pas plus près de l'artisan de la Grèce antique que de l'ouvrier québécois de la fin du XIX ${ }^{\mathbf{e}}$ siècle ?

Certains historiens refusent les modèles en histoire. Tout en acceptant le principe des relations interdisciplinaires, ils craignent toute velléité de systématisation, sous prétexte de perdre une partie de la richesse des sources. Ces historiens, sous le couvert d'une certaine objectivité, ont tendance à oublier qu'ils sont eux-mêmes tributaires de leurs propres catégories mentales plus ou moins conscientes.

Les récents travaux de maîtrise présentés à l'Institut d'histoire de l'Université Laval marquent un moment important de la recherche historique au Québec en même temps qu'un véritable essor de l'histoire sociale du Québec. Leurs auteurs ont ouvert de nouvelles voies de recherche. Sur le plan méthodologique, il importe de souligner l'apport de Jacques Rouillard qui, sans sacrifier à la vérité historique, a su, à la lumière de ses lectures en sociologie, poser des hypothèses et fournir des expli- 
cations inédites. Entre un modèle global d'interprétation et une histoire purement descriptive, il y a place, dans l'état actuel de la recherche, pour l'élaboration d'hypothèses portant sur certains points particuliers de la recherche. En terminant nous croyons utile de signaler certains travaux en préparation qui s'inscrivent dans ce courant de l'histoire sociale.

Ouvrage paru récemment :

Hamelin, Jean,

Paul Larocque et

Jacques Rouillard

Ouvrage en préparation:

En collaboration

Boismenu, Jean

Creval, Jacques

Dickinson, John

Espesset, Hélène

Hardy, Jean-Pierre

Beauchamp, Claude
Répertoire des grèves dans la province de Québec au XIX ${ }^{e}$ siècle. Montréal, Presses de l'Ecole des Hautes Etudes commerciales, 1970. $168 \mathrm{p}$.

Les travailleurs québécois 1851-1896. (Par un groupe d'étudiants gradués sous la direction de M. Jean Hamelin).

Quelques thèses en cours sur l'histoire sociale du Québec:

Coopération et syndicalisme agricole au Québec, 1900-1930, Doctorat (Sociologie), Paris. Tuteur: Henri Desroche. (M. Beauchamp est professeur de sociologie à l'Université Laval).

L'administration fédérale et le problème ouvrier, 1900-1909. M.A. (Histoire), Université de Montréal. Tuteur: Michel Brunet.

L'évolution socio-économique de la Péninsule gaspésienne de 1900 à 1950. Doctorat (Histoire), Université Laval. Tuteur: Yves Roby.

La justice seigneuriale de Notre-Damedes-Anges. M.A. (Histoire), Université Laval. Tuteur: Jacques Mathieu.

Le mouvement ouvrier dans la ville de Québec de 1840 à 1880. Doctorat (Histoire), Université Laval. Tuteur: Jean Hamelin.

Les engagés et les apprentis en Nouvelle-France. M.A. (Histoire), Université Laval. Tuteur: Jacques Mathieu. 
Harvey, Fernand

Linteau, Paul-André

Paquin, Michel

Reid, Philippe

Roberge, Pierre

Ruddell, Terence

Southam, Peter
Les travailleurs québécois et la Commission du travail, 1887-1889. M. Sc.S. (Sociologie), Université Laval. Tuteur: Fernand Dumont.

Histoire de la ville de Maisonneuve 1883-1918. Doctorat (Histoire), Université de Montréal. Tuteurs: Alfred Dubuc et Maurice Séguin.

Etude sur le rôle social des agronomes 1850-1950. M.A. (Histoire), Université Laval. Tuteur: Jean Hamelin.

Etude des premières représentations de l'ensemble de la société québécoise à travers Le Canadien, 1806-1850. (Etude de la bourgeoisie professionnelle de l'époque). Doctorat (Sociologie), Université Laval. Tuteur: Fernand Dumont.

Déterminantes des relations entre mouvements ouvriers et nationalismes dans certaines régions d'industrialisation externe. Doctorat, (Sociologie), Université Laval. Tuteur: André Lux. (Etude comparative entre le Québec, le Canada et divers pays).

Les travailleurs de la ville de Québec, 1790-1840. Doctorat (Histoire), Université Laval. Tuteur: Jean Hamelin.

Evolution du gouvernement municipal au Québec, de 1920 à 1939. Doctorat (Histoire), Université Laval. Tuteur: Jean-Charles Bonenfant. 